\title{
A Rapid Ecological Assessment (REA) Quantitative Survey Method for Benthic Algae Using Photoquadrats with Scuba ${ }^{1}$
}

\author{
Linda B. Preskitt, ${ }^{2}$ Peter S. Vroom, ${ }^{3}$ and Celia M. Smith ${ }^{2}$
}

\begin{abstract}
The challenge of assessing seldom-visited, benthic substrates has created the need for a method to describe benthic communities quickly and efficiently. Macroscale rapid ecological assessments (REAs) of algal assemblages provide managers of coral reefs and other benthic ecosystems with the fundamental descriptive data necessary for continued yearly monitoring studies. The high cost of monitoring marine communities, especially remote sites, coupled with the time limitations imposed by scuba, require that statistically valid data be collected as quickly as possible. A photoquadrat method using a digital camera, computer software for photographic analysis, and minimal data collection in the field was compared with the conventional method of point-intersect (grid) quadrats in estimating percentage cover in subtidal benthic communities. In timed studies, photoquadrats yielded twice the number of quadrats (and an almost infinite number of data points) as conventional methods, provided permanent historical records of each site, and minimized observer bias by having only one observer identifying algae in the field. However, photoquadrats required more post-collection computer analyses of digital photographs than conventional methods. In the manual method, observer bias in algal identification can occur depending on the degree of experience of individual divers. On the other hand, photoquadrats rely on one observer in the field and one observer in the laboratory, standardizing algal identification. Overall, photoquadrats do not yield the finer resolution in diversity that was found using point-intersect quadrats but do provide a more precise estimate of percentage cover of the abundant species, as well as establishing a permanent visual record in the time allowed by work with other teams.
\end{abstract}

EXTENDING OVER $1930 \mathrm{~km}$ northwest of Kaua' $i$ in the main Hawaiian Islands is the Northwestern Hawaiian Islands (NWHI) Coral Reef Ecosystem Reserve: $257,700 \mathrm{~km}^{2}$ of marine reserve that was created by presidential executive order in 2000. Since the

${ }^{1}$ This project was supported by the Botany Department, University of Hawai'i at Mānoa; Hawai'i Coral Reef Initiative (HCRI); and the National Oceanic and Atmospheric Administration. Manuscript accepted 27 May 2003.

2 Department of Botany, University of Hawaici at Mānoa, 3190 Maile Way, Honolulu, Hawai'i 96822.

${ }^{3}$ Coral Reef Ecosystem Investigation, Honolulu Laboratory, National Marine Fisheries Service, 1125B Ala Moana Boulevard, Honolulu, Hawai'i 96814.

Pacific Science (2004), vol. 58, no. 2:201-209

(C) 2004 by University of Hawai'i Press reserve's instatement, the Coral Reef Ecosystem Investigation (U.S. National Marine Fisheries Service) and the Division of Aquatic Resources (Department of Land and Natural Resources, State of Hawai'i) have conducted yearly and biyearly, respectively, assessment and monitoring research expeditions to the numerous islands and reefs situated in this remote part of the Pacific. These trips have included oceanographers and algal, coral, fish, and invertebrate biologists in a cooperative effort to quantitatively assess this isolated and relatively untouched ecosystem. Teams of biologists have conducted surveys to record the flora and fauna using rapid ecological assessment (REA) protocols with scuba. As the name implies time was limited, thus requiring carefully designed methods for quantitative data collection. The goal of the algal surveys was to describe the algal community 
structure and prepare a comprehensive species list for each site. Working at depths of 3 to $16 \mathrm{~m}$ with teams using other rapid assessment methods, we recognized the need for a method of algal assessment that would minimize time in the water yet yield the most statistically sound data.

Conventional methods using point grid, point-intersect methods, or visual estimation have been compared with the use of photographic and video quadrats (Bohnsack 1979, Foster et al. 1991, Meese and Tomich 1992, Roberts et al. 1994) with mixed results. Conventional methods yielded more taxa (Bohnsack 1979, Foster et al. 1991) and could be manipulated to provide data for estimating percentage cover at over $100 \%$ to account for canopy effects. However, these methods were found to be less precise, less accurate (Foster et al. 1991), and more time consuming in the field (Bohnsack 1979, Meese and Tomich 1992, Dethier et al. 1993, Leonard and Clark 1993, Aronson et al. 1994). Photographic and video survey methods were found to be faster and more objective (Bohnsack 1979, Meese and Tomich 1992, Leonard and Clark 1993, Aronson et al. 1994), to decrease variation among observers (Meese and Tomich 1992) but be more time consuming in the laboratory (Meese and Tomich 1992, Leonard and Clark 1993). Some difficulty in identifying smaller individuals was experienced, resulting in a lower taxa resolution (Foster et al. 1991, Roberts et al. 1994). In comparing video with photography, video was less time consuming and provided a better permanent record, but photography provided higher resolution for identifying macroalgae to species (Aronson et al. 1994). Based upon these studies we initially considered photographic quadrats for their timesaving capabilities, better precision and accuracy, less observer variation, and permanent historical records. However, the twodimensionality of the photoquadrat method has been criticized because canopy effects are not considered (Foster et al. 1991) and substrate rugosity in coral reef regions makes laboratory algal identification difficult (Bohnsack 1979, Leonard and Clark 1993, Roberts et al. 1994).

With these considerations, a REA survey method was designed to quantitatively assess algal cover using photoquadrats, collect herbarium vouchers, and map cryptic species. The objective of this study was to analyze the cost/benefit ratio of the photoquadrat method versus the conventional point grid quadrat method often used in field assessments. This study did not attempt to test statistical applications that may be applied to the data gathered with the REA method, but ANOSIM statistical analyses with PRIMER software were done to compare the results of the two methods. The goal of the REA surveys for which this method was devised is to gather quantitative data from which significant changes in overall community structure and levels of abundance can be detected. Therefore, ANOSIM, or analysis of similarities, was used to test for significant differences in the algal community structure where fluctuations in abundance of species or species composition might indicate environmental change. This method also provides flexibility for other statistical applications that are covered in other papers (see Dethier et al. 1993, BenedettiCecchi et al. 1996, Miller and Ambrose 2000; C. Bird, unpubl. data).

\section{MATERIALS AND METHODS}

This comparative study was conducted on 29 November 2002 at Hōnaunau Bay, Hawai'i Island, Hawai'i. The southwest-facing site is well protected from all but southern swells and is well known for large coral formations starting at $3 \mathrm{~m}$ deep and extending well past $30 \mathrm{~m}$. The substrate comprises mainly filamentous algal turfs, the corals Porites spp. and Pavona varians, and macroalgae located in crevices between coral heads. Parallel to shore, two $25-\mathrm{m}$ transects were set $50 \mathrm{~m}$ apart at $10 \mathrm{~m}$ deep. To compare efficiency and data consistency between the two methods, two two-person scuba teams, one team using the photoquadrat method described in this paper and the other using a conventional grid quadrat method, surveyed both transects. The primary purpose was to compare the number of quadrats completed in situ in $30 \mathrm{~min}$ and the consistency of the data between the two methods. 


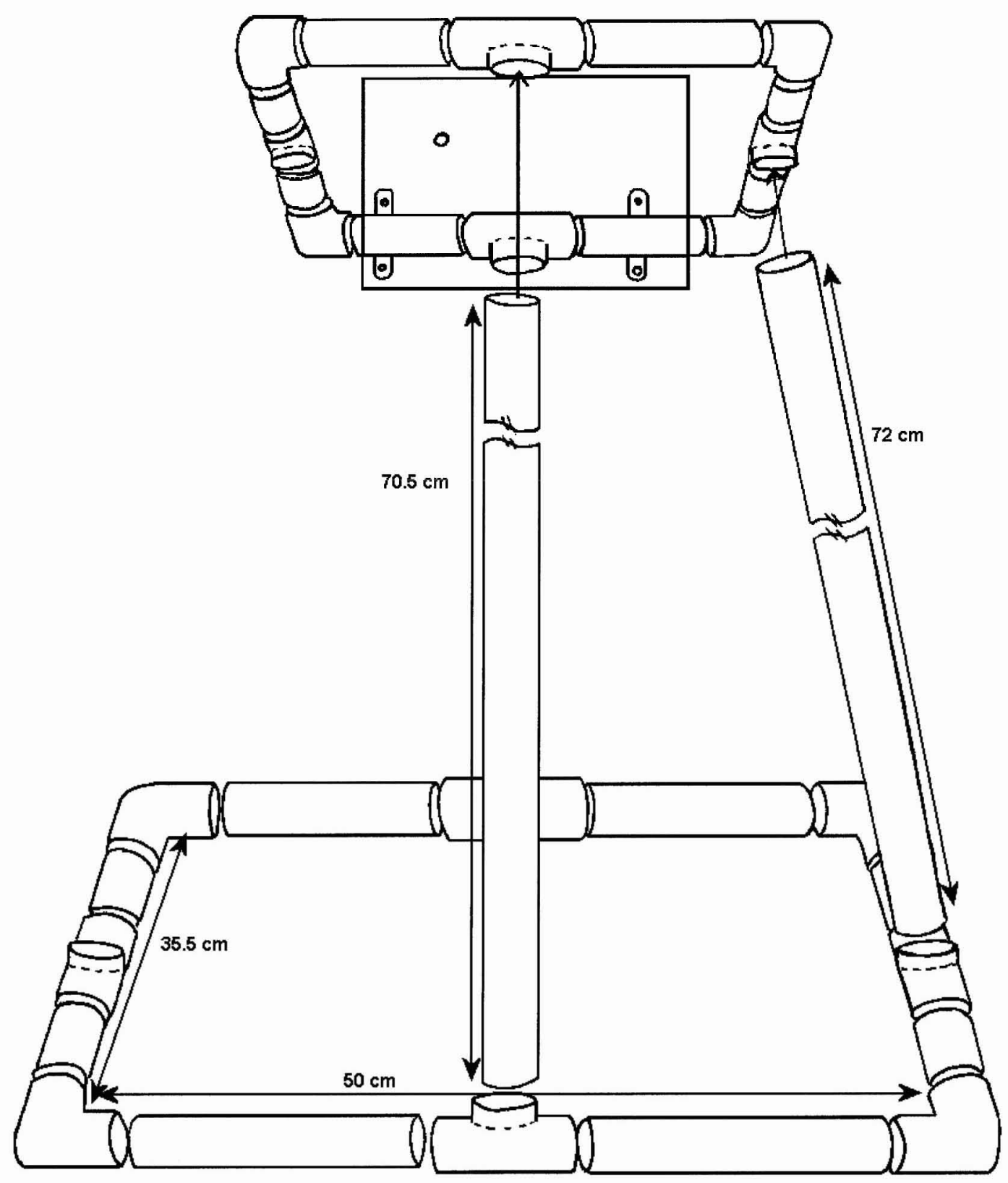

FIGURE 1. PVC photoquadrat framer with dimensions for an Olympus 3030CZ camera in Olympus PT007 housing.

\section{Photoquadrat Method}

A digital camera (Olympus 3030CZ) with a housing (Olympus PT007) was mounted on a $0.18-\mathrm{m}^{2}$ polyvinyl chloride (PVC) photo- quadrat framer (Figure 1). The camera was set at SHQ resolution and forced flash with a digital slave strobe (Ikelite Substrobe DS50) mounted on one leg of the framer. The lower perimeter of the framer was marked with 


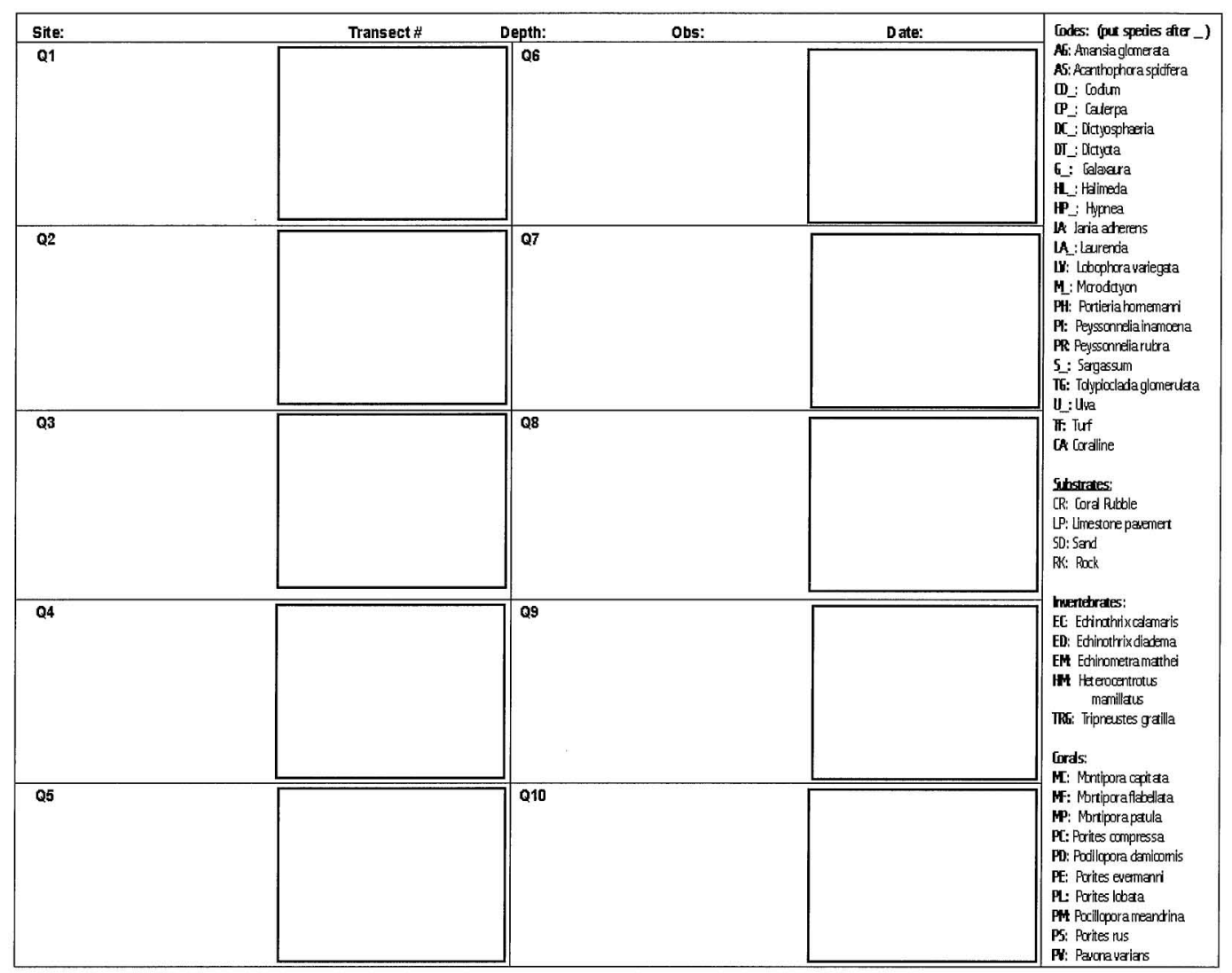

Figure 2. Photoquadrat data sheet with space for observational notes for 10 quadrats. Boxes for drawing maps indicating the location of cryptic species are positioned within each larger quadrat box. A list of codes for common genera and species is on the right-hand side of the data sheet.

quadrat number and centimeter scales to provide location and scale information in each photograph for later computer analysis. The quadrat data were recorded on a waterproof data sheet that included space for recording the species found in the quadrat, a "map" area for identifying cryptic species in depressions that might prove difficult in computer analysis, and codes for the most common genera and species of macroalgae, corals, invertebrates, and substrate types to standardize and shorten note taking (Figure 2).

Two trained observers moved along each transect, with one placing the framer and operating the camera and the other taking notes
(Figure 3). The photoquadrat framer's short edge was placed along the transect with the lower left corner of the quadrat positioned at the meter mark and a picture taken. Before moving the photo quadrat, the second diver identified algae within the photoquadrat, recorded the relative abundance of the five most abundant algae on a scale of 1 to 5 (with 1 being most abundant), drew a quadrat map locating species that may be hard to identify in a photograph, and collected in numbered self-sealing plastic bags representative samples of the algal species in the quadrats from outside the framer for later identification in the laboratory. Once data were recorded, the 


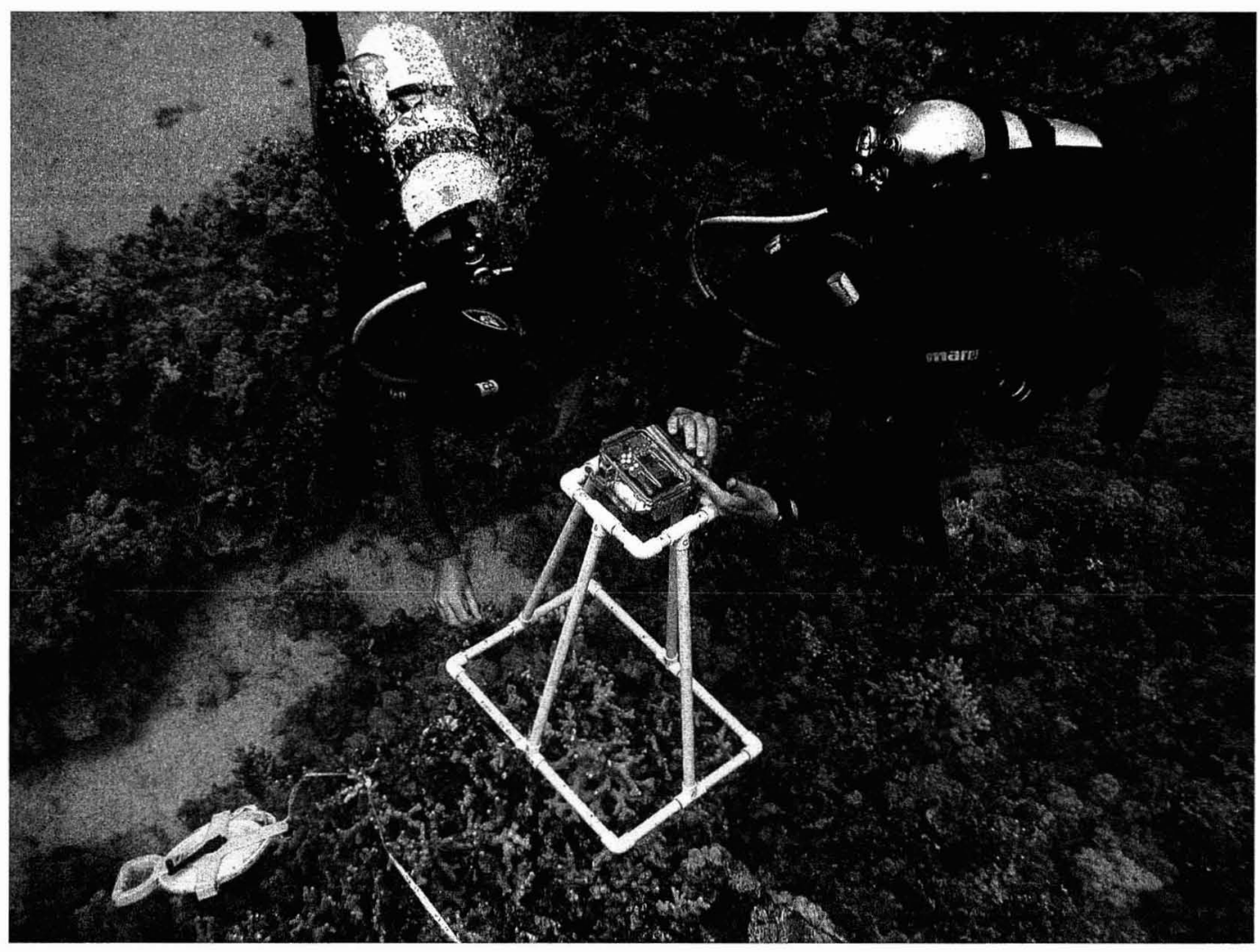

Figure 3. Kim Peyton and Brooke Stuerke using photoquadrat to measure algal cover, Puakō, Hawai'i.

photoquadrat was moved to the next meter mark and the procedure repeated for a total of $30 \mathrm{~min}$. To prevent redundancy, only samples of new algal species found in subsequent quadrats were collected.

The photographs were downloaded to a personal computer, renamed with a unique location code, then cropped and color autocorrected in image-editing software. Each photo was analyzed for percentage cover using PhotoGrid (C. Bird, Department of Botany, University of Hawai ${ }^{i}$ ), a software program capable of random and stratified random point analysis on digital photography. To be comparable with the conventional manual method, 30 random points were generated for the photographic analysis. Once all photos were analyzed, data were imported into a standard spreadsheet for further statistical application.

\section{Manual Quadrat Method}

To be consistent with the photoquadrat method, a $0.18-\mathrm{m}^{2}$ PVC quadrat similar to the photoquadrat frame was double-strung with nylon line to form 49 intersection points in grids on both sides of the quadrat. Thirty points were randomly selected and marked with colored string on the grid. To ensure objectivity and compensate for parallax, an observer lined up the marked intersect on both sides of the grid and recorded the organism or substrate under that particular double intersect. Data sheets included a table for recording the data on each point in each 
TABLE 1

List of Species/Substrate Type and Percentage Cover by Method

\begin{tabular}{lccc}
\hline \hline & & $\%$ Cover \\
\cline { 2 - 4 } Category & All 10 & First 10 \\
Manual Quadrats & Photoquadrats & All \\
Melanamansia glomerata & 0.7 & 0.0 & 0.007 \\
Dictyota ceylanica & 0.0 & 0.0 & 0.002 \\
Lobophora variegata & 0.3 & 0.0 & 0.000 \\
Rhipidosiphon javensis & 0.0 & 0.0 & 0.002 \\
Tolypiocladia glomerulata & 1.0 & 0.035 & 0.028 \\
Crustose coralline & 0.5 & 0.002 & 0.003 \\
Turf & 30.8 & 0.367 & 0.367 \\
Montipora capitata & 0.2 & 0.008 & 0.008 \\
Pavona varians & 14.5 & 0.067 & 0.071 \\
Pocillopora meandrina & 0.2 & 0.002 & 0.001 \\
Porites spp. & 51.3 & 0.515 & 0.469 \\
Unknown coral & 0.0 & 0.002 & 0.001 \\
Sand & 0.0 & 0.002 & 0.040 \\
Sea urchins & 0.5 & 0.002 & 0.001 \\
$\quad$ Total no. of categories & 10 & 10 & 13 \\
\hline
\end{tabular}

Note: ANOSIM testing for similiarity between the two methods resulted in an $R$ value of $0.078 \%(P<1.8 \%)$.

quadrat and the codes for the most common genera and species of macroalgae, corals, invertebrates, and substrate types to standardize the note taking and speed up recording time.

Each diver was assigned 15 random intersect points. The two observers swam together along the transect, one placing the lower left corner of the quadrat on each meter mark in the same location that the photoquadrat had been placed. Each observer then recorded the data of their assigned intersect points and proceeded to the next quadrat, working down the transect for $30 \mathrm{~min}$.

Data were entered into a standard spreadsheet and transformed into percentage cover. An ANOSIM test using PRIMER software (Clarke and Gorley 2001) was conducted on the data to determine if there was a significant difference in similarity between the manual versus photoquadrat methods in the algal community structure.

\section{RESULTS}

Before the camera batteries were depleted, the two-person team using digital photoquadrats completed 22 quadrats on transect 1 in $30 \mathrm{~min}$ and 16 quadrats on transect 2 in 21 min, an average of 1.36 and 1.31 min per quadrat, respectively. The conventional manual method was completed on 10 quadrats on each transect in the allotted $30 \mathrm{~min}$, averaging 3 min per quadrat. Laboratory analysis of the algal samples and the 38 photographs averaged $8 \mathrm{~min}$ per photo to rename, crop, color correct, analyze with random points, identify accompanying algal samples, and export data into a worksheet.

The number of categories (algal species, coral, invertebrate, and substrate type) found in the two methods varied slightly (Table 1). Ten categories were identified in the 20 quadrats of the grid quadrat method and the first 20 photoquadrats. The category list increased to 13 when all 38 photoquadrats were considered. Macroalgal species identified in the grid quadrat method included Melanamansia glomerata, Lobophora variegata, Tolypiocladia glomerulata, turf and red crustose coralline algal forms, and the corals Montipora capitata, Pavona varians, Pocillipora meandrina, and Porites spp. Utilization of all 38 photoquadrats added the algal species Dictyota ceylanica and Rbipidosiphon javensis and a new substrate, sand, to the category list. 
The percentage cover data from both transects, using all 10 quadrats per transect from the manual method and the same first 10 quadrats from the photoquadrat method, were analyzed for similarities using PRIMER software. The data were fourth root-transformed and a one-way ANOSIM was conducted. The analysis resulted in an $R$ value of 0.078 $(P<1.8 \%)$. No differences were found in the community composition or levels of abundance of species between the two methods, or similarities between and within the sites. The near zero $R$ value shows a very high level of similarity between the quadrats from the two methods.

\section{DISCUSSION}

The digital photoquadrat method clearly allowed data to be collected from more quadrats than the conventional point grid quadrat method. Photoquadrats are not new; they have been compared with a myriad of pointintersect, visual estimation, and grid quadrat methods and have often been found wanting in scale and in diversity measurements. This study was an attempt to refine the standard photoquadrat method by adding a two-observer team, note taking, collection of samples, and quadrat mapping to address known concerns with cryptic species. Though not used in this comparison of quantitative methods, the REA protocol includes finishing the dive with a general algal collection of the site in which rare, cryptic, and less-abundant species not present in the photoquadrats were collected for the comprehensive species list. The photoquadrat method described here satisfies the REA protocol requirements and allows algal teams to complete robust sampling measures in the same time periods as the methods for other dominant benthic cover in the NWHI and the main Hawaiian Islands.

Practical advantages of the photoquadrat method over manual quadrats were numerous. The method produced twice as many quadrats (and data) that were as statistically rigorous as the conventional grid quadrat method; quadrats located on vertical walls and pinnacles were more accessible; and little field training and algal identification experience were required for half of the team. The framer is easily modified for closer resolution and smaller organisms, many of which would be too small to sample manually with a small grid quadrat underwater; samples collected provided more accurate identification in the laboratory and doubled as voucher samples for previously unvisited sites; and the photographs provided permanent records available to other researchers for a variety of additional in-depth analyses. Finally, observational bias is lessened with the photoquadrat method. The biyearly trips required six phycologists (three teams of two) in the field simultaneously, necessitating taking less-trained observers for some of the positions. With this photoquadrat method, variation among field observers was decreased because only one team member needed to be trained in macroalgal identification. In addition, observational bias and error were greatly lessened with only one person analyzing digital photographs in the laboratory.

It was clear that limitations exist with the photoquadrat method. Underwater digital equipment is costly and susceptible to flooding or breakage, requiring more monetary investment in equipment and backups than conventional methods. However, such costs are already standard with other organismal assessments. The best photoquadrats were taken on relatively flat surfaces; with greater rugosity comes greater difficulty in the identification of species in crevices and holes on the reef. Adding a digital slave strobe increased the focal point of the camera in these low light conditions and made these dark areas more interpretable in the photographs. In addition, recording which species were most abundant in the field helped when identifying species on the computer screen: matching the most abundant species from field data sheets to the species observed in digital images minimized misidentification. The mapping of quadrats with greater rugosity also increased the level of interpretation of hard-to-see areas in the photographs. However, even with these added steps, rare, very small, and cryptic species were often overlooked in photoquadrats. 
The two-dimensionality of photoquadrats will always prove costly in areas of algal canopy. Excessive canopy completely conceals the understory in two-dimensional photography. Removing biomass and taking a series of pictures (Bohnsack 1979, Foster et al. 1991) is not possible within the REA time limits. However, field notes by divers in areas with dense algal canopies will mark these regions for future research under less-stringent time constraints. Finally, laboratory analyses of samples and photographs can take as much as 8 more minutes per quadrat.

The most noticeable advantage of photoquadrats over grid quadrats was flexibility in statistical analysis. Grid quadrats are static: the number of random points are fixed, usually with too few points due to time limitations, and sometimes the observer must quickly identify algae underwater in less than ideal conditions. In comparison, the photoquadrat method is more flexible in laboratory analysis. The method of data collection on the computer can vary to accommodate investigators' questions. New developments in photographic analysis software (such as PhotoGrid) allow the observer to either determine the style (stratified or not) and number of random points for the calculation of percentage cover or measure actual area for statistical analysis. The added flexibility in number of quadrats, number of points, and number of photographic analysis methods provides more choices in statistical applications of the photoquadrat data. To maximize statistical analyses but minimize effort, power analyses should be conducted to estimate the sampling effort needed to ensure that the study is powerful enough to detect the desired sensitivity to change.

Ecological assessment and monitoring methods are powered by the purpose of the project. If fine taxa resolution and an in-depth description of an algal community including canopy effect are desired, then twodimensional photoquadrats will not suffice. However, the objectives of REAs are broader. The goal of an REA is to quantitatively describe an ecosystem at a macro-community level. The photoquadrat method provides adequate quantitative data, analysis flexibility, and permanent algal specimens that enable the investigators to determine the patterns in distribution and abundance of macroalgae and turfs in these remote, inaccessible regions. A standardized REA protocol not only provides the quantitative data needed to establish baselines for these communities but also ensures that comparable data are collected during the ongoing monitoring needed for management decisions.

\section{ACKNOWLEDGMENTS}

We thank Ryan Okano, Kim Page, Kim Peyton, Cheryl Squair, and Koa Shultz for field testing the photoquadrat; Jen Smith and the Marine Macrophytes seminar group for their critical comments and encouragement; and Chris Bird for his statistical insight and use of his PhotoGrid program.

\section{Literature Cited}

Aronson, R. B., P. J. Edmunds, W. F. Precht, D. W. Swanson, and D. R. Levitan. 1994. Large-scale, long-term monitoring of $\mathrm{Ca}$ ribbean coral reefs: Simple, quick, inexpensive techniques. Atoll Res. Bull. 421: $1-19$.

Benedetti-Cecchi, L., L. Airoldi, M. Abbiati, and F. Cinelli. 1996. Estimating the abundance of benthic invertebrates: A comparison of procedures and variability between observers. Mar. Ecol. Prog. Ser. 138:93101.

Bohnsack, J. A. 1979. Photographic quantitative sampling of hard-bottom benthic communities. Bull. Mar. Sci. 29 (2): 242252.

Clarke, K. R., and R. N. Gorley. 2001. PRIMER-E Ltd. Plymouth Marine Laboratory, Plymouth, U.K.

Dethier, M. N., E. S. Graham, S. Cohen, and L. M. Tear. 1993. Visual versus randompoint percent cover estimations: 'Objective' is not always better. Mar. Ecol. Prog. Ser. 96:93-100.

Foster, M. S., C. Harrold, and D. D. Hardin. 1991. Point vs. quadrat estimates of the cover of sessile marine organisms. J. Exp. Mar. Biol. Ecol. 146:193-203. 
Leonard, G. H., and R. P. Clark. 1993. Point quadrat versus video transect estimates of the cover of benthic red algae. Mar. Ecol. Prog. Ser. 101:203-208.

Meese, R. J., and P. A. Tomich. 1992. Dots on the rocks: A comparison of percent cover estimation methods. J. Exp. Mar. Biol. Ecol. 165:59-73.

Miller, A. W., and R. F. Ambrose. 2000.
Sampling patchy distributions: Comparison of sampling designs in rocky intertidal habitats. Mar. Ecol. Prog. Ser. 196:114.

Roberts, D. E., S. R. Fitzhenry, and S. J. Kennelly. 1994. Quantifying subtidal macrobenthic assemblages on hard substrata using a jump camera method. J. Exp. Mar. Biol. Ecol. 177:157-170. 